\title{
Postoperative Coronary Artery Spasm After Mitral Valve Replacement and Cox- Maze IV Procedure: A Case Report
}

\author{
Qianlei Lang, MD,${ }^{1 *}$ Chunyan Li, BSMed,${ }^{2 *}$ Chaoyi Qin, MD,${ }^{1}$ Wei Meng, $\mathrm{MD}^{1}$ \\ ${ }^{1}$ Department of Cardiovascular Surgery, West China Hospital, Sichuan University, Chengdu, P.R. China; \\ ${ }^{2}$ Department of Operation Management, West China Hospital, Sichuan University, Chengdu, P.R. China
}

\section{ABSTRACT}

Background: Few cases have been reported about coronary artery spasm after a mitral valve replacement and concomitant Cox-Maze IV procedure. We report the case of an adult male who developed right coronary artery (RCA) spasm after a mitral valve replacement with tricuspid valve repair and Cox-Maze IV procedure.

Case report: A 66-year-old male, complaining of progressive exertional shortness of breath, was diagnosed with severe mitral stenosis, moderate tricuspid regurgitation, complete right bundle branch block, and persistent atrial fibrillation $(\mathrm{AF})$ in our clinic. The patient underwent elective mitral valve replacement, tricuspid valve repair, and Cox-Maze IV procedure. Four hours after surgery, a 12-lead electrocardiogram (ECG) showed progressive elevation of ST-segment in the avF and III leads and Troponin-T was over $7000 \mathrm{pg} /$ $\mathrm{mL}$. After one hour, Troponin-T increased to over $10000 \mathrm{pg} /$ $\mathrm{mL}$, and ECG still showed persisted ST-segment elevation in inferior leads. Emergent angiography was performed, and intra-coronary administration of nitroglycerin completely relieved the spasm.

Conclusion: Potential risks of coronary injury after valvular surgery and Cox-Maze IV procedure need further aggressive investigation and postoperative ischemia should prompt an emergent coronary angiography to identify the cause and apply immediate therapy.

\section{INTRODUCTION}

In the three decades since the first Cox-Maze procedure was performed in 1987, surgical treatment for patients with $\mathrm{AF}$ has seen extensive advances. Cox-Maze IV was more frequently performed as a concomitant procedure during other

Received May 30, 2021; received in revised form fune 16, 2021; accepted fune 16, 2021.

*These two authors contribute equally to this article.

Correspondence: Wei Meng, MD, Department of Cardiovascular Surgery, West China Hospital/West China School of Medicine, Sichuan University, 610007, Chengdu, P.R. China, 37 GuoXue Alley, Chengdu, Sichuan, P.R. China, Telephone +86028 85422897, Fax +86028 85421833 (e-mail: meng_wei_1111@yahoo.com). cardiac surgeries, especially involving mitral valve diseases. It is reported that radiofrequency catheter ablation was related to coronary arteries, including spasm, strictures, and stenosis [Hishikari 2014]. However, few cases have been reported about coronary artery spasm after a mitral valve replacement and concomitant Cox-Maze IV procedure for persistent AF. We report the case of an adult male who developed RCA spasm after a mitral valve replacement with tricuspid valve repair and Cox-Maze IV procedure.

\section{CASE REPORT}

A 66-year-old male, complaining of progressive exertional shortness of breath, was diagnosed with severe mitral stenosis, moderate tricuspid regurgitation, complete right bundle branch block, and persistent AF in our clinic. The patient underwent elective mitral valve replacement, tricuspid valve repair, and Cox-Maze IV procedure using the Cardioblate ${ }^{\circledR}$ LP system (Medtronic, Minneapolis, Minnesota, USA).

Preoperative angiography showed mild stenosis of RCA and left anterior descending artery, and transthoracic echocardiography showed deteriorated left ventricular function with $40 \%$ ejection fraction.

The surgery was uneventful and total aortic clamp time was $82 \mathrm{~min}$. Cardioplegia was perfused through the aortic root every 15-20 min, and the core temperature was cooled down to $34^{\circ} \mathrm{C}$. During the removal of cardiopulmonary bypass, the ECG monitor showed a 90-100 bpm sinus rhythm alternated with a pacing rhythm of $80 \mathrm{bpm}$.

The patient was transferred to our intensive care unit (ICU) with junctional rhythm. Four hours after surgery, a 12-lead ECG showed progressive elevation of ST-segment in the avF and III leads, and Troponin-T was over $7000 \mathrm{pg} / \mathrm{mL}$. Therefore, acute myocardial infarction of the inferior wall was highly suspected. Given that myocardial injury of cardiopulmonary bypass and radiofrequency ablation, and stable hemodynamic condition, cath lab was informed and a recheck of Troponin-T was arranged. After one hour, Troponin-T increased to over $10000 \mathrm{pg} / \mathrm{mL}$, and ECG still showed persisted ST-segment elevation in inferior leads (Figure 1 A-D). (Figure 1) Although hemodynamic was stable and transthoracic echocardiography showed acceptable left ventricular contraction, emergent angiography was performed in the cath lab. Distal part of RCA spasm was found, and intracoronary administration of nitroglycerin completely relieved 


\section{A. post-operative in ICU}

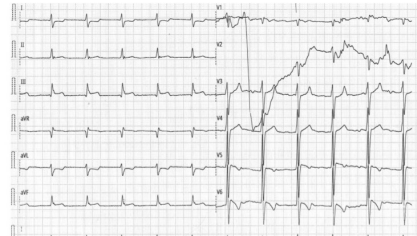

\section{C. post-operative $5 \mathrm{hrs}$ in ICU}
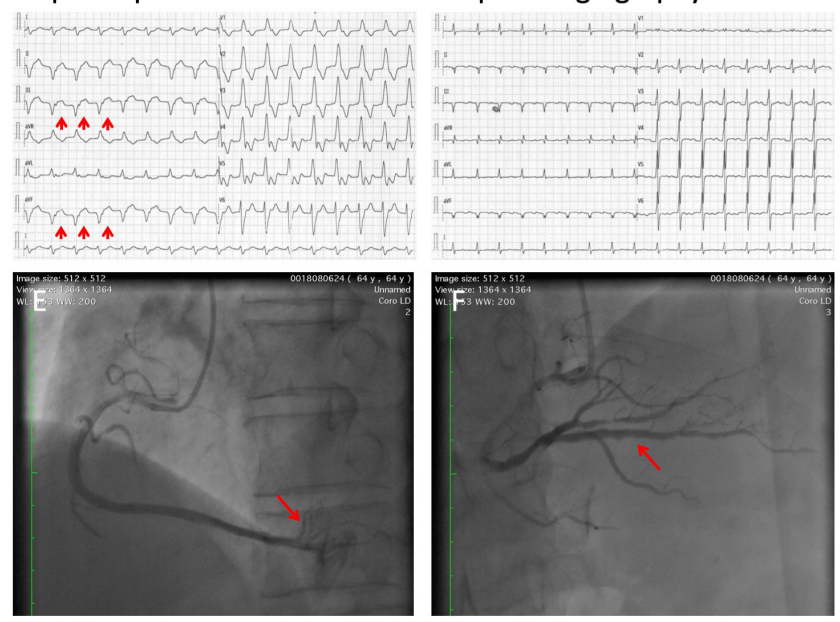

Figure 1. Dynamic variants of ECG and angiography images. (A) Immediate 12-leads ECG was performed when the patient was transferred into our ICU and showed junctional rhythm. (B, C) 4 hours after surgery, ECG showed elevation of ST-segment of inferior leads and 1 hour later, progressive elevation of inferior leads was identified (arrows). (D) After emergent angiography and intracoronary administration of nitroglycerin, ECG showed normal sinus rhythm. (E) Emergent angiography showed distal part of right coronary spasm (arrows). (F) Intracoronary administration of nitroglycerin relieved the spasm (arrows) and angiography showed only mild stenosis of distal part of right coronary artery, which was as the same as the preoperative angiography.

the spasm (Figure $1 \mathrm{E}-\mathrm{F}$; Supplementary video 1 and 2). The patient was maintained on nitrates and aspirin after full discovery. No recurrent elevation of ST-segment and Troponin$\mathrm{T}$ was recorded.

\section{DISCUSSION}

Radiofrequency surgical ablation was commonly used for the Cox-Maze procedure, and many studies approved its safety and efficacy. Permanent pacemaker implantation, bleeding, and myocardial injury were common complications after the Cox-Maze procedure. Coronary artery spasm after valvular surgery and concomitant Cox-Maze IV procedure rarely was reported.

Some studies reported severe coronary artery spasm after catheter radiofrequency ablation. It is well known that direct thermal damage from radiofrequency energy or an imbalance in autonomic nervous system activity might be responsible. It is reported that atrium contained epicardial ganglionated plexuses consist of different patterns of sympathetic and

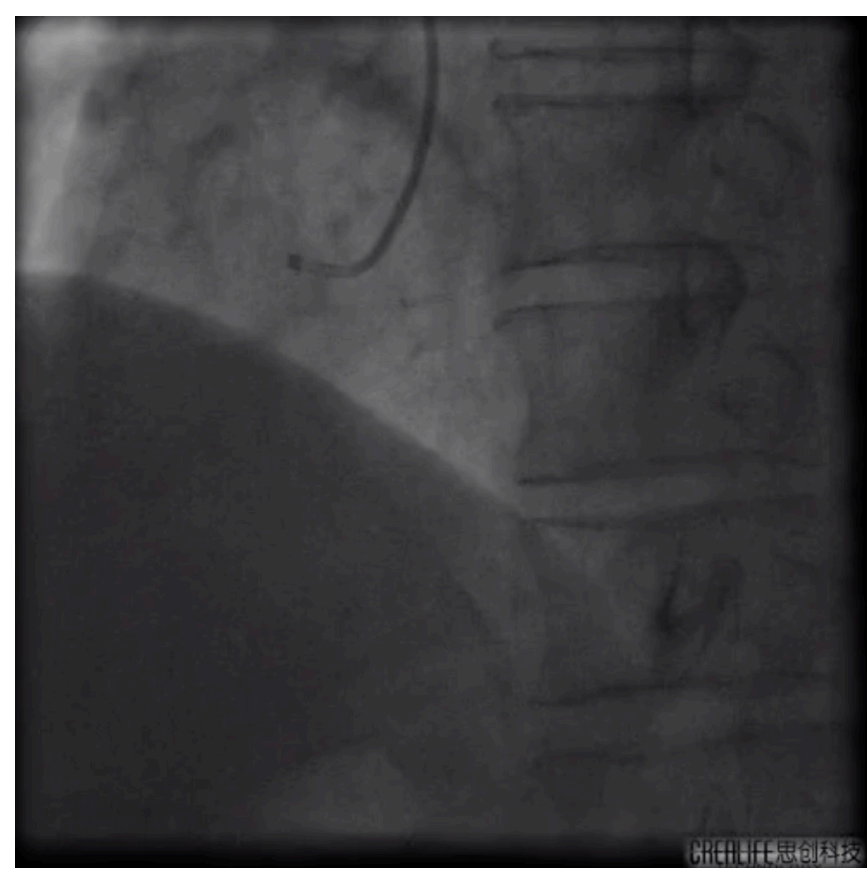

Supplementary video 1. Pre-treatment angiography. Emergent angiography showed distal part of right coronary spasm. (https://vimeo. com/580410566)

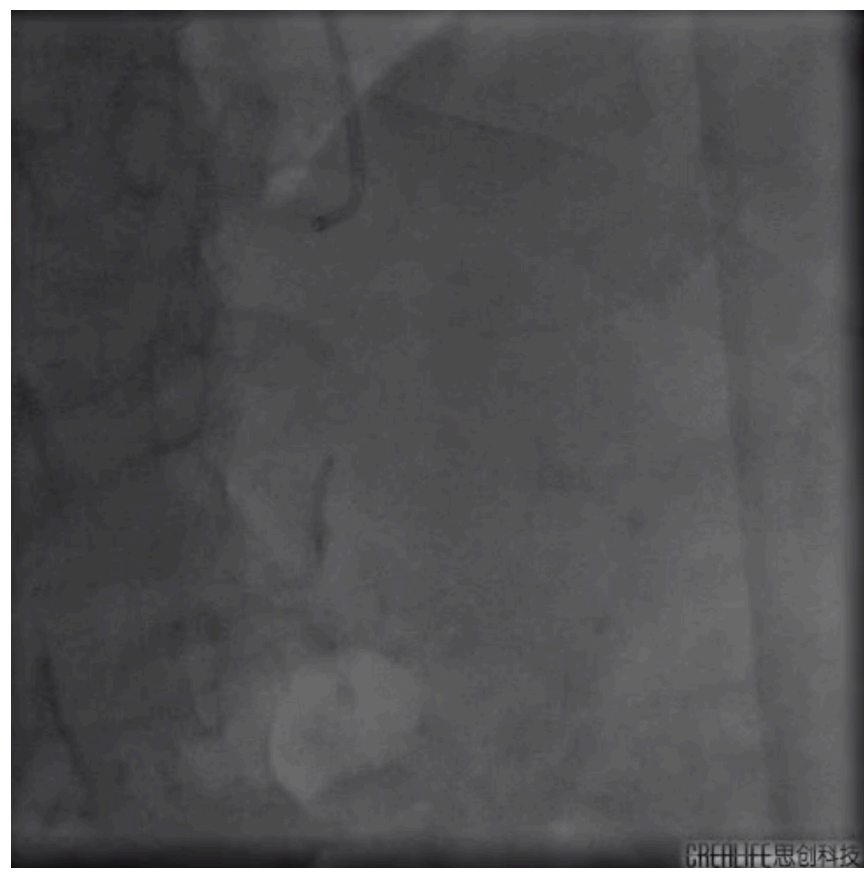

Supplementary video 2. Post-treatment angiography. Intracoronary administration of nitroglycerin relieved the spasm and angiography showed only mild stenosis of distal part of right coronary artery, which was as the same as the preoperative angiography. (https://vimeo.com/580411563) 
vague nerves, which could cause an imbalance in autonomic nervous system activity under different stress [Nakagawa 2009]. In addition, complete resolution of coronary artery spasm through intracoronary infusion of nitroglycerin helped to rule out air embolism or thrombosis.

The actual incidence of postoperative coronary artery spasm basically was unknown. In an English literature review, nearly $20 \%$ of cases were only suspected as a result of the clinical presentation without coronary angiography [Pragliola 2015]. In accordance with other reports that showed the most common postoperative coronary artery spasm happened in the ICU up to six hours after surgery, our patient presented with elevations of ST-segment and Troponin-T at four hours after the surgery. Given that preoperative angiography showed mild stenosis of both the right and left coronary artery, intensive oxidative stress, inflammation and changes of vascular reactivity might result in the postoperative coronary artery spasm [Ruel 2004]. In the present case report, coronary spasm relief with establishment of q waves may be related to the time of cardiopulmonary bypass and myocardial ischemia-reperfusion injury.

Postoperative coronary artery spasm can be a lethal event, and early mortality was high [Pragliola 2015]. However, once angiography was conducted, direct intracoronary administration of nitrates or calcium channel blockers could be rapidly effective.

\section{CONCLUSION}

In conclusion, potential risks of coronary injury after valvular surgery and Cox-Maze IV procedure need further aggressive investigation, and postoperative ischemia should prompt an emergent coronary angiography to identify the cause and apply immediate therapy.

\section{ACKNOWLEDGEMENT}

Written informed consent of clinical detail and image publication was obtained from the patient.

\section{REFERENCES}

Hishikari K, Kuwahara T, Takahashi A, Isobe M. 2014. Severe coronary artery spasm during radiofrequency ablation for atrial fibrillation. Int J Cardiol. 172:e513-5.

Nakagawa H, Scherlag BJ, Patterson E, Ikeda A, Lockwood D, Jackman WM. 2009. Pathophysiologic basis of autonomic ganglionated plexus ablation in patients with atrial fibrillation. Heart Rhythm. 6:S26-34.

Pragliola C, Gaudino M, Farina P, Massetti M. 2015. Postoperative coronary artery spasm after mitral valve replacement. Int J Surg Case Rep. 8C:185-8.

Ruel M, Khan TA, Voisine P, Bianchi C, Sellke FW. 2004. Vasomotor dysfunction after cardiac surgery. Eur J Cardiothorac Surg. 26:1002-14. 